\title{
On New Quasi-Type-2 Fuzzy Logic Systems
}

\author{
Jerry M. Mendel, Life Fellow, IEEE, and Feilong Liu, Student Member, IEEE
}

\begin{abstract}
This paper provides an answer to the question that the type-2 fuzzy logic community is now asking: "What comes after interval type-2 fuzzy logic systems (IT2 FLSs)?" It demonstrates, through a geometrical understanding of the type-reduced set, that logical next steps in the progression from type-1 to interval type-2 to type-2 FLSs are quasi-T2 FLSs, either an interconnection of a T1 FLS and an IT2 FLS, or an interconnection of two IT2 FLSs, in which both FLSs are designed simultaneously. The quasi-T2 FLSs overcome the computational difficulties that are associated with set theoretic operations and type-reduction (TR) for general T2 FSs and FLSs, because all set theoretic operations can be performed as in existing T1 or IT2 FLSs, and because TR for an IT2 FLS can be performed using existing KM Algorithms.
\end{abstract}

\section{INTRODUCTION}

$\mathrm{T}$ he type-2 (T2) fuzzy logic (FL) community is now asking: "What comes after interval type-2 fuzzy logic systems (IT2 FLSs)?" Some suggest immediately developing a full-blown T2 FLS [3], [4], [7]. Others suggest first developing a restricted T2 FLS in which all of the secondary membership functions (MFs) are symmetrical triangles [25]. While it is very tempting to immediately develop a full-blown T2 FLS, to do so is fraught with computational difficulties (e.g., how to compute set theoretic and arithmetic operations and perform type-reduction (TR) for general T2 fuzzy sets (FSs) are active areas of research [1], [5]-[7], [12], [14], [20], [26]). Even developing a restricted T2 FLS has similar problems, although approximations for set theoretic operations and TR are possible. This raises the following question: "Is there a rational way to decide between developing a full-blown T2 FLS and a restricted T2 FLS as the next step in the hierarchy of FLSs, from a type-1 (T1) to an IT2 to a T2 FLS?" This paper provides a basis for answering this question, and proposes neither the full-blown nor restricted T2 FLSs as the next step. Instead, it proposes quasi-T2 FLSs (defined in Section VII) based on a new geometric understanding of TR for a T2 FS.

\section{WIDELY-USED REPRESENTATIONS OF A T2 FS}

A type-2 fuzzy set (T2 FS) $\tilde{A}$ can be represented in different ways [16]. The point-valued representation (which is usually the starting point for understanding or describing a general T2 FS) is one in which the membership function

Jerry M. Mendel and Feilong Liu are with the Signal and Image Processing Institute, Ming Hsieh Department of Electrical Engineering, University of Southern California, Los Angeles, CA 90089-2564, USA (phone: 213-740-4445; fax: 213-740-4651; email: mendel@sipi.usc.edu, feilong1@usc.edu)
(MF) of $\tilde{A}$ is specified at every point in its $2 \mathrm{D}$ domain of support, i.e.

$$
\tilde{A}=\left\{\left((x, u), \mu_{\tilde{A}}(x, u)\right) \mid \forall x \in X, \forall u \in J_{x} \subseteq[0,1]\right\}
$$

The vertical slice representation [29], [23], [24] focuses on each value of the primary variable $x$, and expresses (1) as:

$$
\begin{gathered}
\tilde{A}=\int_{x \in X} \mu_{\tilde{A}}(x) / x \\
\mu_{\tilde{A}}(x)=\int_{u \in J_{x} \subseteq[0,1]} f_{x}(u) / u
\end{gathered}
$$

$\mu_{\tilde{A}}(x)$ is called a secondary $M F$ or a vertical slice, and $f_{x}(u)$ is called the secondary grade. The vertical slice representation is extremely useful for computation and may also be useful for theoretical studies.

The wavy-slice representation [18] is one in which $\tilde{A}$ is represented as the union of all of its embedded T2 FSs. An embedded T2 FS is a T2 FS that has only one primary membership at each $x_{i}$, and is also called a wavy slice [18]. For discrete (or sampled) universes of discourse, this means:

$$
\tilde{A}=\sum_{j=1}^{n_{A}} \tilde{A}_{e}^{j}
$$

where $n_{A}$ is the number of embedded T2 FSs, $\tilde{A}_{e}^{j}$. This representation of a T2 FS, in terms of much simpler embedded T2 FSs, is not recommended for computational purposes, because it would require the explicit enumeration of the $n_{A}$ embedded T2 FSs and $n_{A}$ can be astronomical; however, it is very valuable in theoretical studies because it quickly leads to the structure of the solution to a new problem, after which practical procedures are determined to compute the solution.

\section{ALPHA-LEVEL REPRESENTATION OF A T2 FS}

A very valuable representation of a T1 FS uses $\alpha$-cuts [11], but to-date, a comparable kind of representation has not been widely used for T2 FSs. Such a representation would use horizontal slices of a T2 FS, which would be planes called $\alpha$-planes so that this representation would be called the $\alpha$-level representation. Note that this representation for a T2 FS has already appeared in the literature [2], [26] and we are not claiming credit for it $^{1}$. The following definition of an $\alpha$-plane for a T2 FS is a generalization of an $\alpha$-cut for a T1 FS.

Definition 1. An $\alpha$-plane for a general T2 FS $\tilde{A}$, denoted $\tilde{A}_{\alpha}$, is the union of all primary memberships of $\tilde{A}$ whose

\footnotetext{
${ }^{1}$ It also appears in the unpublished report [12].
} 
Proceedings of 2008 International Conference on Fuzzy Systems (FUZZ 2008), WCCI 2008,

Hong Kong, CHINA, June 1-6, 2008

secondary grades are greater than or equal to $\alpha(0 \leq \alpha \leq 1), \quad$ is two-dimensional. (3) Similar to the properties of an $\alpha$-cut i.e.

$$
\begin{aligned}
& \tilde{A}_{\alpha}=\bigcup_{x \in X}\left\{(x, u) \mid \mu_{\tilde{A}}(x, u) \geq \alpha\right\}=\bigcup_{x \in X} S_{\tilde{A}}(x \mid \alpha) . \\
& S_{\tilde{A}}(x \mid \alpha) \equiv\left(\mu_{\tilde{A}}(x)\right)_{\alpha}=\left[s_{L}(x \mid \alpha), s_{R}(x \mid \alpha)\right]
\end{aligned}
$$

$S_{\tilde{A}}(x \mid \alpha)$ is the $\alpha$-cut of the secondary MF $\mu_{\tilde{A}}(x)$ [recall that $\mu_{\tilde{A}}(x)$ is a T1 FS].

Example 1. Fig. 1 depicts a vertical-slice representation for $\tilde{A}$, i.e. at each sampled value of primary variable $x$ the secondary MF, $\mu_{\tilde{A}}(x)$, is shown. The diagram in Fig. 1 is easy to draw and gives the appearance of a $3 \mathrm{D}$ diagram, but it is on a $2 \mathrm{D}$ plane, which is why it is called a " $2 \frac{1}{2}-\mathrm{D}$ representation." An example of an $\alpha$-plane for this $\tilde{A}$ is given as the dark gray shaded area in Fig. 2, where the continuous primary variable $x$ is sampled as shown. Each of the dark black vertical lines corresponds to $S_{\tilde{A}}\left(x_{i} \mid \alpha\right)$.

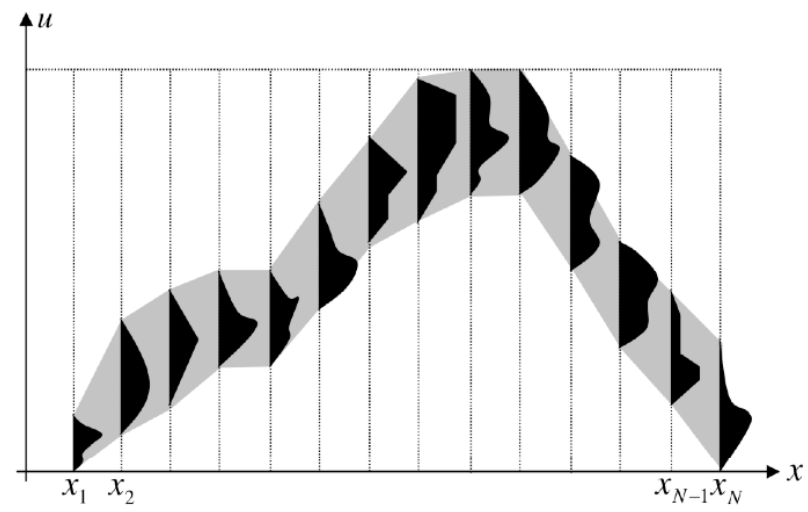

Figure 1. $2 \frac{1}{2}-\mathrm{D}$ representation of the $3 \mathrm{D}$ MF for $\mu_{\tilde{A}}(x, u)$. The gray shaded area is the FOU. The black filled-in entities are secondary MFs $\mu_{\tilde{A}}\left(x_{i}\right)$. They are filled in only for the effect, and give the $2 \mathrm{D}$ figure a $3 \mathrm{D}$ effect.

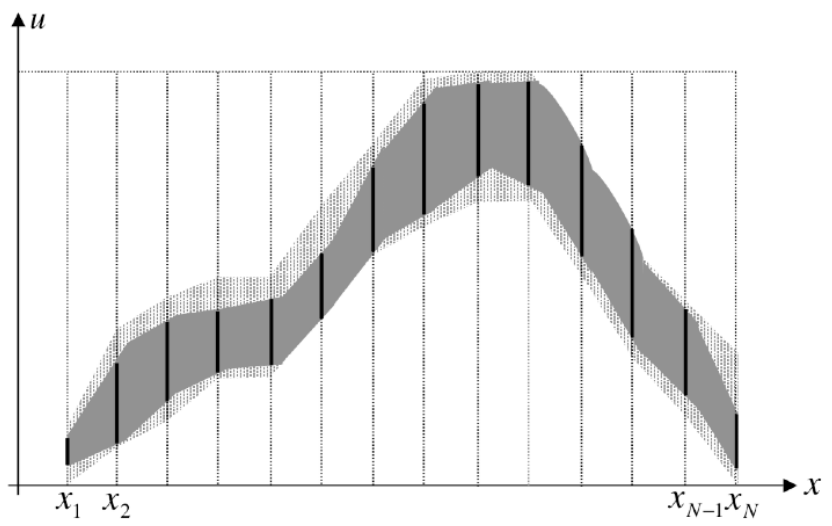

Figure 2. The black vertical lines are $\alpha$-cuts for the Fig. 1 secondary MFs. They all fall within the FOU (the light filled-in area). The dark gray shaded area just connects the $\alpha$-cuts, and is only shown here to make the visualization look more interesting.

Comments: (1) The 2D domain of $\tilde{A}$, for continuous universes of discourse is called the footprint of uncertainty of $\tilde{A}, \operatorname{FOU}(\tilde{A})$, and it is the $\alpha=0$ plane. (2) Compared to an $\alpha$-cut, which is a one-dimensional interval, an $\alpha$-plane for a T1 FS, one can show: [12]: (a) $\tilde{A}_{\alpha_{1}} \subseteq \tilde{A}_{\alpha_{2}}$ if $\alpha_{1} \geq \alpha_{2}$; (b) $(\tilde{A} \cup \tilde{B})_{\alpha}=\tilde{A}_{\alpha} \cup \tilde{B}_{\alpha}$; and, (c) $(\tilde{A} \cap \tilde{B})_{\alpha}=\tilde{A}_{\alpha} \cap \tilde{B}_{\alpha}$.

Definition 2. Let $I_{\tilde{A}_{\alpha}}(x, u \mid \alpha)$ be the following $3 \mathrm{D}$ indicator function for $\alpha$-plane $\tilde{A}_{\alpha}$ :

$I_{\tilde{A}_{\alpha}}(x, u \mid \alpha)=\left\{\begin{array}{ll}1 & (x, u) \in \tilde{A}_{\alpha} \\ 0 & (x, u) \notin \tilde{A}_{\alpha}\end{array}, x \in X\right.$ and $u \in S_{\tilde{A}}(x \mid \alpha)$

Associated with $I_{\tilde{A}_{\alpha}}(x, u \mid \alpha)$ is the following $\operatorname{FOU}\left(\tilde{A}_{\alpha}\right)$, called an $\alpha$-level FOU:

$$
\operatorname{FOU}\left(\tilde{A}_{\alpha}\right)=\alpha I_{\tilde{A}_{\alpha}}(x, u \mid \alpha)
$$

$\operatorname{FOU}\left(\tilde{A}_{\alpha}\right)$ is the $\alpha$-plane, $\tilde{A}_{\alpha}$, raised off of the $x-u$ plane up to the level $\alpha$. An example of $\operatorname{FOU}\left(\tilde{A}_{\alpha}\right)$ for the example in Figs. 1 and 2 is depicted in Fig. 3.

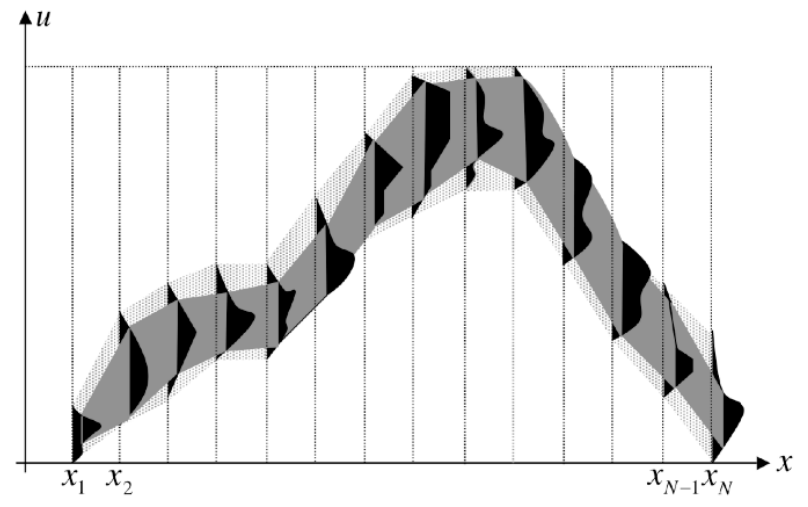

Figure 3. The dark gray shaded area is $\operatorname{FOU}\left(\tilde{A}_{\alpha}\right)$ for the example in Fig. 2.

Theorem 1. ( $\alpha$-Level Representation). A general T2 FS $\tilde{A}$ can be represented as the union of its $\alpha$-level FOUs, i.e.

$$
\tilde{A}=\bigcup_{\alpha \in[0,1]} \operatorname{FOU}\left(\tilde{A}_{\alpha}\right)
$$

\section{CENTROID TYPE-REDUCTION}

\section{A. Type-Reduction (TR)}

In a T2 FLS the most intensive computation is TR. We shall use TR to answer the earlier question: "Is there a rational way to decide between developing a full-blown T2 FLS and a restricted T2 FLS as the next step in the hierarchy of FLSs?"

Definition 3: Type-reduction is a collection of operations that map a function of T2 fired-rule output fuzzy sets into a T1 FS.

There are different functions that can be used for TR, and to-date five kinds of TR have been described: centroid, center of sums, height, modified height and center of sets [16], [10]. Because of space limitations, this paper focuses only on centroid TR, but its results apply to all TR methods (see [20] for the other kinds of TR).

\section{B. Centroid TR}


For centroid TR, all fired-rule output T2 FSs, $\tilde{B}^{l}$, are first combined by finding their union, i.e.

$$
\bigcup_{l=1}^{M} \tilde{B}^{l} \equiv \tilde{B}
$$

where

$$
\mu_{\tilde{B}}(y)=\sqcup_{l=1}^{M} \mu_{\tilde{B}^{\prime}}(y) \quad \forall y \in Y
$$

in which $\mu_{\tilde{B}^{\prime}}(y)$ is the secondary MF for the $t^{\text {th }}$ rule, and the rectangular symbol denotes the join operation. How to compute $\mu_{\tilde{B}^{\prime}}(y)$ is not needed in this paper (see, e.g. [16], [4], [6]).

As an application of the wavy slice representation given in (4), the centroid TR set, $Y_{c}(\mathbf{x})$, is obtained as the union of the centroids of all the embedded T2 FSs of $\tilde{B}$, i.e.

$$
Y_{c}\left(\mathbf{x}^{\prime}\right)=\int_{\theta_{1} \in J_{y_{1}}} \cdots \int_{\theta_{N} \in J_{y_{N}}} \min \left(f_{y_{1}}\left(\theta_{1}\right), \ldots, f_{y_{N}}\left(\theta_{N}\right)\right) / \frac{\sum_{i=1}^{N} y_{i} \theta_{i}}{\sum_{i=1}^{N} \theta_{i}}
$$

In this equation $\theta_{i}, J_{y_{i}}$ and $f_{y_{i}}(\forall i)$ are associated with $\mu_{\tilde{B}}(y)$ and $Y_{c}\left(\mathbf{x}^{\prime}\right)$ is shown as an explicit function of $\mathbf{x}=\mathbf{x}^{\prime}$ because each $\mu_{\tilde{B}^{\prime}}(y)$ in (11) is a function of FLS input $\mathbf{x}$. For different FLS inputs, different values of $Y_{c}\left(\mathbf{x}^{\prime}\right)$ are obtained.

Until very recently, the only way to compute $Y_{c}\left(\mathbf{x}^{\prime}\right)$ was to use the following (brute-force) procedure [16]. For each $\mathbf{x}=\mathbf{x}^{\prime}$ :

(1) Compute $\mu_{\tilde{B}}(y)$ using (11);

(2) Discretize the $y$-domain into $N$ points $y_{1}, \ldots, y_{N}$, as in Fig. 1;

(3) Discretize each $J_{y_{i}}\left(\mathbf{x}^{\prime}\right)=\left[\underline{\mu}_{\tilde{B}}\left(y_{i}\right), \bar{\mu}_{\tilde{B}}\left(y_{i}\right)\right]$ [the primary memberships of $\mu_{\tilde{B}}(y)$ at $\left.y_{i}\right]$ into a suitable number of points, say $M_{i}(i=1, \ldots, N)$, and let $\theta_{i}\left(\mathbf{x}^{\prime}\right) \in J_{y_{i}}\left(\mathbf{x}^{\prime}\right)$

(4) Enumerate all the embedded $\mathrm{T} 1$ sets of $\tilde{B}$; there will be $\prod_{i=1}^{N} M_{i}$ of them; and,

(5) Compute the centroid of each enumerated embedded $\mathrm{T} 1$ set and assign it a membership grade equal to the minimum of the secondary grades corresponding to that enumerated embedded $\mathrm{T} 1$ set.

The procedure just described is impractical for computation because it requires the explicit enumeration of the embedded T2 FSs and $\prod_{i=1}^{N} M_{i}$ centroid calculations, and, this number will in general be astronomical, which is why it is referred to it as a "brute-force procedure." Hence, if centroid TR is to be used for general T2 FSs, a practical computational procedure must be found.

\section{Recent Works on Centroid TR}

A recent approach for computing $Y_{c}(\mathbf{x})$, based on randomly sampling embedded T2 FSs and computing their centroids [7] claims to give rise to a significant reduction in the time or resources needed to perform type-reduction. A theoretical explanation for this has not yet appeared.

Coupland [3] has suggested that TR be bypassed if in fact only a defuzzified value is desired. To that end, he recommends the use of the $x$-coordinate of the geometric centroid of the 3D MF of $\mu_{\tilde{B}}(y)$ in (11). This approach does not reduce to T1 defuzzified results when all sources of uncertainty disappear; hence, comparing its results with all earlier results cannot be done on a level playing field.

Lucas et al. [14] have proposed first computing the centroids of vertical slices and then computing the centroid of the resulting function. Although ad hoc, this is a rather novel idea.

\section{New Way to Perform Centroid TR}

Each $\alpha$-plane, $\tilde{A}_{\alpha}$, can be interpreted as an IT2 FS, so each $\alpha$-plane has a centroid, $C_{\tilde{A}_{\alpha}}(y)$ (recall that the centroid of an IT2 FS is an interval-valued set that is completely described by its two end-points [9], [16]), i.e.

$$
C_{\tilde{A}_{\alpha}}(y)=\left[l_{\tilde{A}_{\alpha}}, r_{\tilde{A}_{\alpha}}\right]
$$

Let $I_{\bar{C}_{\bar{\alpha}_{\alpha}}}(y \mid \alpha)$ denote the following indicator function associated with $C_{\tilde{A}_{\alpha}}(y)$ :

$$
I_{C_{\tilde{A}_{\alpha}}}(y \mid \alpha)= \begin{cases}1 & y \in C_{\tilde{A}_{\alpha}}(y) \\ 0 & y \notin C_{\tilde{A}_{\alpha}}(y)\end{cases}
$$

Theorem 2. [12], [20] The centroid type-reduced set for a general T2 FS $\tilde{A}, Y_{c}\left(\mathbf{x}^{\prime}\right)$, is the following T1 FS:

$$
Y_{c}\left(\mathbf{x}^{\prime}\right)=\bigcup_{\alpha \in[0.1]} \alpha I_{C_{\tilde{A}_{\alpha}}}(y \mid \alpha)
$$

Comments: (1) The proof of this theorem is in [20]. (2) Each centroid, $C_{\tilde{A}_{\alpha}}(y)$ has the structure of a fuzzy weighted average (FWA) (e.g., see [13]); (3) $C_{\tilde{A}_{\alpha}}(y)$, can be computed using KM Algorithms [13]; (4) If $m \alpha$-cuts are used, then the $2 m$ end-point computations for the $m$ centroids can all be performed in parallel; and, (5) As shown in Section V, $Y_{c}\left(\mathbf{x}^{\prime}\right)$ does not have to be computed in order to understand its geometrical properties.

\section{ObSERVATIONS About the Shape of a CENTRoid}

The $\alpha$-plane decomposition of the T2 FS $\tilde{B}$ provides a very valuable way to think about the shape of the centroid of $\tilde{B}$, and in this section it is shown that this shape is predictable and consequently that the computation of the centroid can be greatly simplified.

To begin, note that each $\alpha$-level FOU, $\operatorname{FOU}\left(\tilde{B}_{\alpha}\right)$, can be associated with an interval T2 FS whose secondary grade equals $\alpha$. Although it is not possible to obtain a closed-form formula for $C_{\tilde{B}_{\alpha}}(y)$, Mendel and $\mathrm{Wu}$ [21], [22] have obtained closed-form uncertainty bound formulas for the two end-points of $C_{\tilde{B}_{\alpha}}(y)$ that show the length of $C_{\tilde{B}_{\alpha}}(y)$ 
increases (decreases) as the area of $\operatorname{FOU}\left(\tilde{B}_{\alpha}\right)$ increases (decreases). Use of this fact is made below.

In the rest of this section, it is assumed that all secondary MFs of $\tilde{B}$ are monotonically non-decreasing for $u_{y} \in\left[0, m_{y}\right]$, monotonically non-increasing for $u_{y} \in\left[m_{y}, 1\right]$, and normal. The support of a secondary MF is $u_{y} \in\left[a_{y}, b_{y}\right]$ where $0 \leq a_{y} \leq m_{y}$ and $m_{y} \leq b_{y} \leq 1$. It may also happen that $\mu_{\tilde{B}}(y)=1$ for $m_{1 y} \leq m_{y} \leq m_{2 y}$. The secondary MFs that are depicted in Fig. 1 have these properties. Other examples of such secondary MFs are triangles and trapezoids.

Based on viewing $\tilde{B}$ in terms of its $\alpha$-planes, it is possible to draw the following conclusions about T1 FS $C_{\tilde{B}}(y)$ :

(1) Maximum uncertainty about $\tilde{B}$ occurs for its $\alpha=0$ plane, $\tilde{B}_{\alpha=0}$;

(2) Minimum uncertainty about $\tilde{B}$ occurs for its $\alpha=1$ plane, $\tilde{B}_{\alpha=1}$

(3) When $\alpha_{i} \geq \alpha_{j}$, then $C_{\tilde{B}_{\alpha_{i}}}(y) \subseteq C_{\tilde{B}_{\alpha_{j}}}(y)$;

(4) $C_{\tilde{B}}(y)$ is first non-decreasing and then non-increasing;

(5) When all secondary MFs are normal at exactly one point, then $\tilde{B}_{\alpha=1}$ is a function (i.e., not a plane) so that $C_{\tilde{B}_{1}}(y)$ is a single point;

(6) When some or all the secondary MFs are trapezoids, then $\tilde{B}_{\alpha=1}$ is a plane and $C_{\tilde{B}_{1}}(y)$ is an interval;

(7) When all secondary MFs are (normal) triangles, then $C_{\tilde{B}}(y)$ is triangle-looking. Its base is computed as $C_{\tilde{B}_{0}}(y)$ and its apex is computed as $C_{\tilde{B}_{1}}(y)$. The latter will be a single point (see Item 5), however its sides may not be straight lines, which is why $C_{\tilde{B}}(y)$ is called "triangle-looking;" and,

(8) When some or all of the secondary MFs are (normal) trapezoids, then $C_{\tilde{B}}(y)$ is trapezoid-looking. Its base is computed as $C_{\tilde{B}_{0}}(y)$ and its top is computed as $C_{\tilde{B}_{1}}(y)$. The latter will be an interval (see Item 6), however, its two sides may not be straight lines, which is why $C_{\tilde{B}}(y)$ is called "trapezoid-looking."

Next, consider a general T2 FS that is totally symmetrical.

Definition 4: A general T2 FS $\tilde{B}$ is said to be totally symmetrical if (a) $\operatorname{FOU}(\tilde{B})$ is symmetrical about primary variable $y$ at $y=m$, and (b) all of its secondary MFs are symmetrical.

The most widely used symmetrical secondary MFs to-date are triangles, since they seem to represent the next logical extension from interval T2 FSs to full-blown general T2 FSs [25]. An example of such a totally symmetrical T2 FS is depicted in Fig. 4.

Theorem 3. If $\tilde{B}$ is totally symmetrical, then the centroid of $\tilde{B}, C_{\tilde{B}}(y)$, is symmetrical about $y=m$, and the average value (i.e., the defuzzified value) of $C_{\tilde{B}}(y)$ equals $m$.

This theorem, whose proof is in [20], is a generalization of similar results obtained in [17] for an IT2 FS. It demonstrates that if one is planning only to use the defuzzified value of a totally symmetrical T2 FS, then to carry out $\mathrm{T} 2$ computations is a wasted effort, because the same result could have been obtained using $\mathrm{T} 1$ calculations.

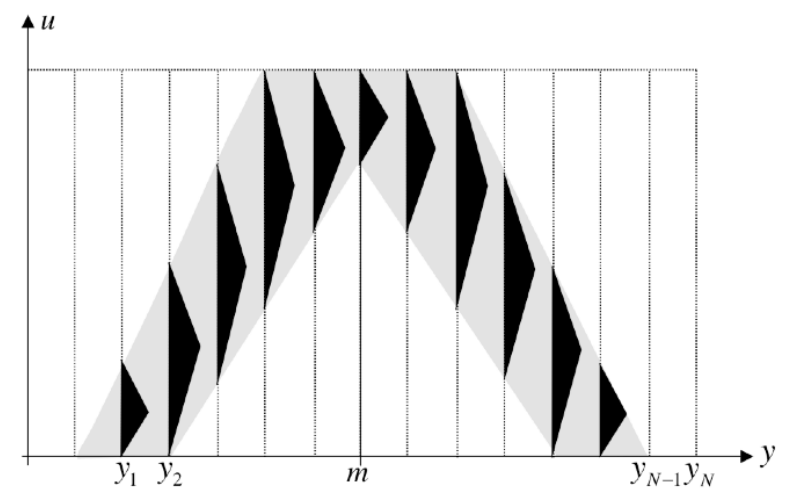

Figure 4. Totally symmetrical T2 FS, whose FOU has a trapezoidal UMF and a triangular LMF, and whose secondary MFs are symmetrical triangles.

\section{SIMULATIONS}

In this section some simulation results are provided that not only support the observations made in Section V but also suggest how to drastically reduce the computations of the centroid.

In the simulations:

(1) Theorem 2 is used as the computational method; it requires first determining $\alpha$-planes and then using the FWA [13];

(2) All of the results are for centroid TR; however, they apply as well to other kinds of TR;

(3) T2 FS $\tilde{B}$ can be thought of as the union of two firedrule output T2 FSs, whose resulting FOU is lower and upper bounded by triangular functions, and whose vertical slices are triangular MFs;

(4) The domain of the primary variable for $\tilde{B}$ is assumed to be $x \in[0,10]$, and to perform centroid TR, $x$ is uniformly sampled using 10,000 samples, such that $x_{i+1}-x_{i}=0.001$;

(5) For $\alpha$-cuts, $\alpha$ is also uniformly sampled by choosing $\Delta=100$ such that $\alpha$ is divided into $\Delta+1$ values, namely $0,1 / \Delta, 2 / \Delta, \ldots,(\Delta-1) / \Delta, 1$; and,

(6) The simulations are for piecewise linear LMF and $\mathrm{UMF}$ and triangle secondary MFs. As shown in Fig. 5a, $\operatorname{UMF}(F O U(\tilde{B}))$ and $\operatorname{LMF}(F O U(\tilde{B}))$ are each the maximum of two piecewise linear functions, i.e.

$$
\begin{aligned}
U M F_{F O U(\tilde{B})}(x)=\max & \left\{\left[\begin{array}{lc}
(x-1) / 2 & 1 \leq x \leq 3 \\
(7-x) / 4 & 3 \leq x \leq 7 \\
0 & \text { otherwise }
\end{array}\right],\right. \\
& {\left.\left[\begin{array}{ll}
(x-2) / 5 & 2 \leq x \leq 6 \\
(16-2 x) / 5 & 6 \leq x \leq 8 \\
0 & \text { otherwise }
\end{array}\right]\right\} }
\end{aligned}
$$


Proceedings of 2008 International Conference on Fuzzy Systems (FUZZ 2008), WCCI 2008,

Hong Kong, CHINA, June 1-6, 2008

$$
\begin{aligned}
& L M F_{F O U(\tilde{B})}(x)=\max \left\{\begin{array}{ll}
(x-1) / 6 & 1 \leq x \leq 4 \\
(7-x) / 6 & 4 \leq x \leq 7 \\
0 & \text { otherwise }
\end{array}\right] \text {, } \\
& \left.\left[\begin{array}{lc}
(x-3) / 6 & 3 \leq x \leq 5 \\
(8-x) / 9 & 5 \leq x \leq 8 \\
0 & \text { otherwise }
\end{array}\right]\right\}
\end{aligned}
$$

Each secondary MF is a triangle, whose base equals $U M F_{F O U(\tilde{B})}(x)-L M F_{F O U(\tilde{B})}(x)$, and whose apex, $\operatorname{Apex}(x)$ is parameterized, for the purposes of this example, as:

$$
\begin{aligned}
\operatorname{Apex}(x)= & L M F_{F O U(\tilde{B})}(x) \\
& +w\left[U M F_{F O U(\tilde{B})}(x)-L M F_{F O U(\tilde{B})}(x)\right]
\end{aligned}
$$

where $w=0,0.25,0.5,0.75,1$. An example of such a secondary MF is depicted in Fig. 5b when $x=2$ and $w=0$. As $w$ varies from 0 to 1 , the triangle changes its shape from a right triangle with its right angle on $L M F_{F O U(\tilde{B})}(x)$, to a non-symmetrical triangle, to a symmetrical triangle, to another nonsymmetrical triangle, and finally to a right triangle with its right angle on $U M F_{F O U(\tilde{B})}(x)$.

The centroid TR sets for the five values of $w$ are depicted in Fig. 6. Surprisingly, each of these TR sets looks symmetrical. Note that when $\alpha=0$, $C_{\tilde{B}_{\alpha=0}}(x)=[3.6605,4.9917]$ (this is the support of all five TR sets), and mean $\left(C_{\tilde{B}_{\alpha=0}}(x)\right) \equiv m\left(C_{\tilde{B}_{\alpha=0}}(x)\right)=4.3261$.

Table I provides numerical details for the five values of $w$. First it provides the apex, $C_{\tilde{B}_{\alpha=1}}(x)$, of each of the "trianglelooking" TR sets. Then it provides the centroid of each TR set, $C_{T R}$ (i.e., the defuzzified value). Observe that $m\left(C_{\tilde{B}_{\alpha=0}}(x)\right)-C_{T R}$ is smaller than 0.0352 for all five values of $w$.

Each TR set was also approximated by a triangle that connected its $\alpha=0$ and $\alpha=1$ values. Table I also provides the centroid ${ }^{2}$ of each approximate TR set, $C_{A T R}$, as well as $C_{A T R}-C_{T R}$. Observe that $C_{A T R}-C_{T R}$ for all five values of $w$ is smaller than 0.0154 .

This example demonstrates that a very good approximation to a centroid type-reduced set can be obtained by computing centroids for only two $\alpha$-cuts, the $\alpha=0$ and $\alpha=1 \quad \alpha$-cuts . See [20] for a second example that supports this conclusion.

The following conclusions can be drawn from the results of these simulations:

(1) Conclusions 1-5 and 7 made in Section V are satisfied, e.g., (Conclusion 4) $C_{\tilde{B}}$ first is non-decreasing and then is non-increasing (see Fig. 6);

2 Centroid of triangle $(l, m, r)$ [left vertex at $(l, 0)$, apex at $(m, h)$ and right vertex at $(r, 0)]: C_{\text {Triangle }}=\frac{1}{3}(m+l+r)$. This formula can be used for any triangle, i.e. it does not have to be shifted to the origin).
(2) Surprisingly, the TR sets are close to symmetrical (see Fig. 6 and Table I); and,

(3) An excellent approximation to a centroid TR set can be obtained using only two $\alpha$-cuts, when this approximation is used to obtain a defuzzified value

\begin{tabular}{|c|c|c|c|c|c|}
\hline & $C_{\tilde{B}_{\alpha=1}}$ & $\begin{array}{c}\text { Centroid } \\
\text { of TR } \\
\text { set, } \\
C_{T R}\end{array}$ & $\begin{array}{l}m\left(C_{\tilde{B}_{\alpha=0}}\right) \\
-C_{T R}\end{array}$ & $\begin{array}{c}\text { Centroid of } \\
\text { Approximate } \\
\text { TR set, } \\
C_{A T R}\end{array}$ & $\begin{array}{l}C_{A T R} \\
-C_{T R}\end{array}$ \\
\hline$w=0$ & 4.3433 & 4.3373 & -0.0112 & 4.3318 & 0.0055 \\
\hline$w=1 / 4$ & 4.3340 & 4.3303 & -0.0042 & 4.3287 & 0.0016 \\
\hline$w=0.5$ & 4.3208 & 4.3213 & 0.0048 & 4.3243 & 0.0030 \\
\hline$w=3 / 4$ & 4.3008 & 4.3090 & 0.0171 & 4.3177 & 0.0087 \\
\hline$w=1$ & 4.2667 & 4.2909 & 0.0352 & 4.3063 & 0.0154 \\
\hline
\end{tabular}
for the T2 FS.

TABLE I. Simulation Results. Note that $m\left(C_{\tilde{B}_{\alpha=0}}(x)\right)=4.3261$.

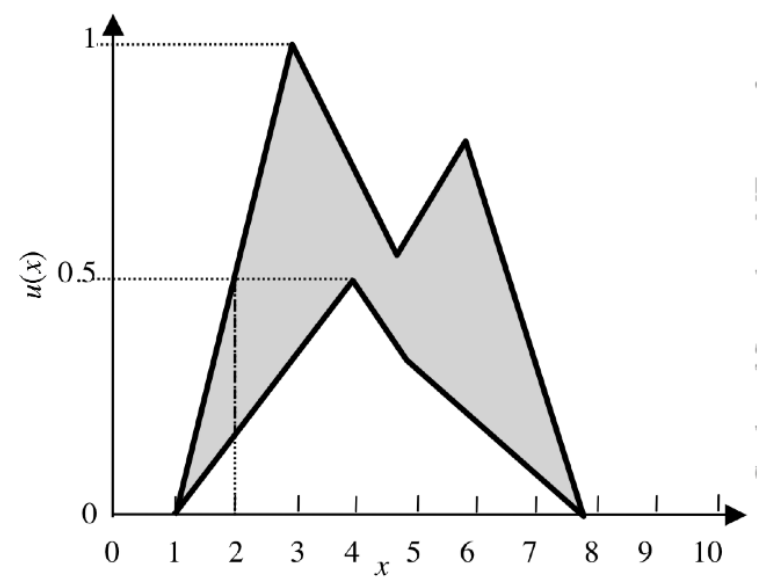

(a)

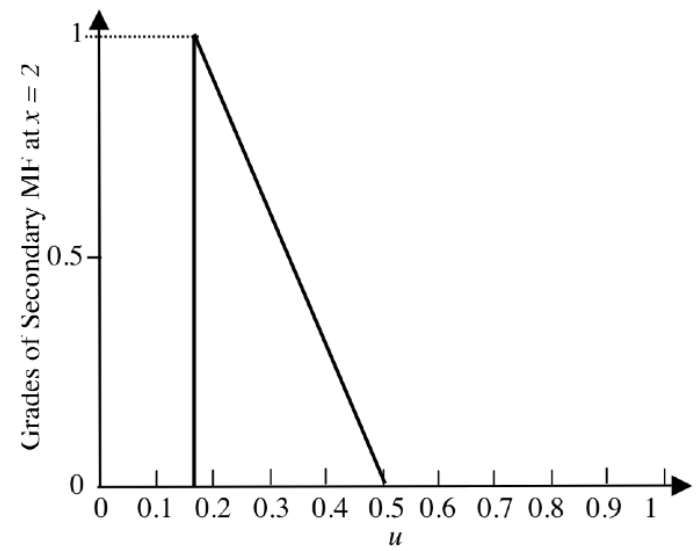

(b)

Figure 5. (a) FOU for simulations, and (b) secondary MF at $x=2$ when $w=0$. 


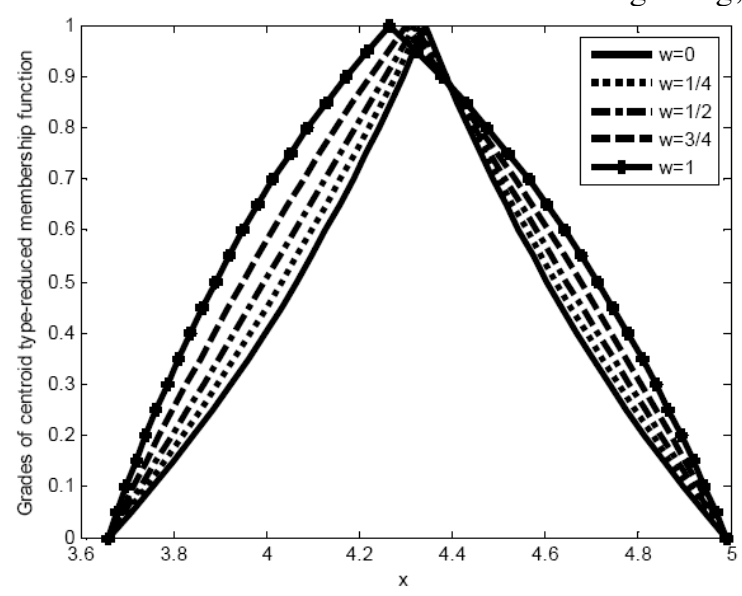

Figure 6. $C_{\tilde{B}}(x)$ for the example when $w=0,0.25,0.5,0.75,1$

\section{PROPOSED NEW ARCHITECTURES FOR T2 FLSS}

Based on the observations made in Sections V and VI, we propose two new architectures for a T2 FLS, as depicted in Fig. 7. The architecture in Fig. 7a is for triangle secondary MFs, whereas the architecture in Fig. $7 \mathrm{~b}$ is for trapezoidal secondary MFs. Both architectures could be called a two $\alpha$-plane T2 FLS or a quasi-T2 FLS. We prefer the latter. Both architectures are much simpler than a full-blown T2 FLS, because they do not involve any T2 calculations. Quasi-T2 FLSs may be good next step in the hierarchy of FLSs, from T1 to IT2 to T2.

The architecture in Fig. 7a is a T2 FLS that combines a T1 FLS and an IT2 FLS. The defuzzified output of the T1 FLS and the TR output of the IT2 FLS provide three points (the vertices of a non-symmetrical triangle), and the overall output of this quasi-T2 FLS is the centroid of that triangle, i.e.

$$
y(\mathbf{x})=\left[y_{L}(\mathbf{x} \mid \alpha=0)+y_{R}(\mathbf{x} \mid \alpha=0)+y^{1}(\mathbf{x} \mid \alpha=1)\right] / 3
$$

The architecture in Fig. 7b is a T2 FLS that combines two IT2 FLSs. The TR outputs of both IT2 FLSs provide four points (the vertices of a non-symmetrical trapezoid), and the overall output of this quasi-T2 FLS is the centroid of that trapezoid, i.e.

$$
\begin{gathered}
y(\mathbf{x})=(2 a+b) / 3(a+b) \\
a=y_{R}(\mathbf{x} \mid \alpha=1)-y_{L}(\mathbf{x} \mid \alpha=1) \\
b=y_{R}(\mathbf{x} \mid \alpha=0)-y_{L}(\mathbf{x} \mid \alpha=0)
\end{gathered}
$$

In both architectures, the MF parameters of the two FLSs are optimized simultaneously, and because of (20) or (21) this is a coupled optimization and design. Because of more design degree of freedoms than are present in only one FLS, we conjecture that the quasi-T2 FLSs have the potential to outperform an IT2 FLS.

TR for an IT2 FLS uses the iterative KM algorithms, so this may cause a computational bottleneck for real-time applications of the quasi-T2 FLSs. A way around this is to replace each of the TR steps in the two quasi-T2 FLSs by Wu-Mendel minimax) uncertainty bounds. Using these bounds directly as an architecture for an IT2 FLS has already been done in [8] and [15], where it has been demonstrated that very good performance can be achieved by doing this.

Much work remains to be done regarding these new quasiT2 FLSs, and we hope that they will be the bases for new studies by the T2 FL community.

\section{CONCLUSIONS}

This paper provides an answer to the question that the T2 FL community is now asking: "What comes after IT2 FLSs?" It demonstrates, through a rigorous understanding of type-reduction for a T2 FS, by means of the alpha-level representation of a T2 FS, and a subsequent geometrical understanding of the type-reduced set, that logical next steps in the progression from T1 to IT2 to T2 FLSs are quasi-T2 FLSs, an interconnection of either a T1 FLS and an IT2 FLS, or an interconnection of two IT2 FLSs. in which both FLSs are designed simultaneously. The quasi-T2 FLSs overcome the computational difficulties that are associated with set theoretic operations and TR for general T2 FSs and FLSs, because all set theoretic operations are those for existing T1 or IT2 FLSs, and because TR for an IT2 FLS can be performed using existing KM Algorithms [9], [16], [19], or even the faster Enhanced KM Algorithms [27]. It is even possible to bypass TR by using the minimax uncertainty bounds in [28], after which it is easy to obtain a very good approximation to the TR set [8], [15]. Applications of the proposed quasi-T2 FLSs remain to be studied.

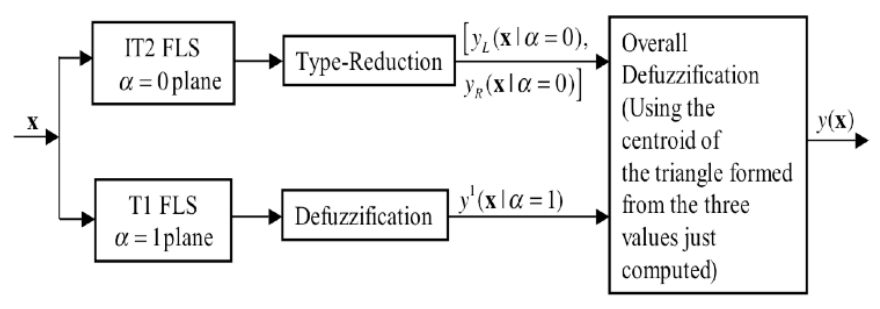

(a)

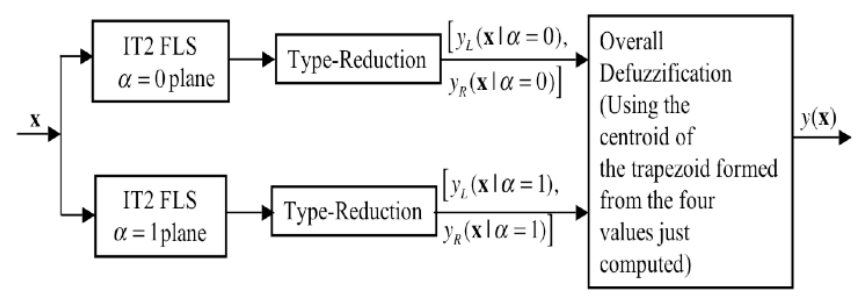

(b)

Figure 7. New architectures for a T2 FLS: (a) Triangle secondary MFs and (b) Trapezoidal secondary MFs.

\section{REFERENCES}

[1] W. Blewitt, S.-M. Zhou and S. Coupland, "A novel approach to type-2 fuzzy addition,” Proc. FUZZ-IEEE, pp. 1456-1461, London, UK, 2007.

[2] Q. Chen and S. Kawase, "On fuzzy-valued fuzzy reasoning," Fuzzy Sets and Systems, Vol. 113, pp. 237-251, 2000.

[3] S. Coupland, "Type-2 fuzzy sets: geometric defuzzification and typereduction," Proc. of IEEE Symposium on Foundations of Computational Intelligence (FOCI 2007), pp. 622-629, Honolulu, HI, April 2007.

[4] S. Coupland and R. I. John, "Geometric type-1 and type-2 fuzzy logic 
systems," IEEE Trans. on Fuzzy Systems, vol. 15, pp. 3-15, February 2007.

[5] S. Coupland and R. I. John, "On the accuracy of type-2 fuzzy sets," Proc. FUZZ-IEEE, pp. 131-136, London, UK, 2007.

[6] S. Greenfield and R. I. John, "Optimized generalized type-2 join and meet operations," Proc. FUZZ-IEEE, pp. 141-146, London, UK, 2007.

[7] S. Greenfield, R. I. John and S. Coupland, "A novel sampling method for type-2 defuzzification," Proc. UKCI 2005, London, September 2005.

[8] H. Hagras, "Type-2 FLCs: a new generation of fuzzy controllers," IEEE Computational Intelligence Magazine, vol. 2, pp. 30-43, Feb. 2007.

[9] N. N. Karnik and J. M. Mendel, "Centroid of a type-2 fuzzy set," Information Sciences, vol. 132, pp. 195-220, 2001.

[10] N. N. Karnik, J. M. Mendel and Q. Liang "Type-2 Fuzzy Logic Systems," IEEE Trans. on Fuzzy Systems, vol. 7, pp. 643-658, Dec. 1999.

[11] G. J. Klir and B. Yuan, Fuzzy Sets and Fuzzy Logic: Theory and Applications, Prentice-Hall, Upper Saddle River, NJ, 1995.

[12] F. Liu, "An efficient centroid type reduction strategy for general type-2 fuzzy logic systems," Walter J. Karplus Summer Research Grant Report, IEEE Computational intelligence Society, 2006.

[13] F. Liu and J. M. Mendel, "Aggregation using the fuzzy weighted average, as computed by the KM algorithms," accepted for publication in IEEE Trans. on Fuzzy Systems, 2007.

[14] L. A. Lucas, T. M. Centeno and M. R. Delgado, "General type-2 inference systems: analysis, design and computational aspects," Proc. FUZZ-IEEE, pp. 1107-1112, London, UK, 2007.

[15] C. Lynch, H. Hagras and V. Callaghan, "Using uncertainty bounds in the design of embedded real-time type-2 neuro-fuzzy speed controller for marine diesel engines. Proc. FUZZ-IEEE, Vancouver, CA, pp. 7217-7224, July 2006.

[16] J. M. Mendel, Uncertain Rule-Based Fuzzy Logic Systems: Introduction and New Directions, Prentice-Hall, Upper-Saddle River, NJ, 2001.

[17] J. M. Mendel, "On a 50\% savings in the computation of the centroid of a symmetrical interval type-2 fuzzy set," Information Sciences, vol. 172,pp. 417-430, 2005.
[18] J. M. Mendel and R. I. John, "Type-2 fuzzy sets made simple," IEEE Trans. on Fuzzy Systems, vol. 10, pp. 117-127, April 2002.

[19] J. M. Mendel and F. Liu, "Super-exponential convergence of the Karnik-Mendel algorithms for computing the centroid of an interval type-2 fuzzy set," IEEE Trans on Fuzzy Systems, vol. 15, pp. 309-320, April 2007

[20] J. M. Mendel and F. Liu, "Alpha-level representation for type-2 fuzzy sets: theory and applications," submitted for publication, 2007.

[21] J. M. Mendel and $\mathrm{H}$. Wu, "Type-2 fuzzistics for symmetric interval type-2 fuzzy sets: forward problems," IEEE Trans. on Fuzzy Systems, vol. 14, pp. 781-792, December 2006.

[22] J. M. Mendel and H. Wu, "Type-2 fuzzistics for non-symmetric interval type-2 fuzzy sets: forward problems," IEEE Trans. on Fuzzy Systems, vol. 15, pp. 916-930, October 2007.

[23] M. Mizumoto and K. Tanaka, "Some properties of fuzzy sets of type2," Information and Control, vol. 31, pp. 312-340, 1976.

[24] M. Mizumoto and K. Tanaka, "Fuzzy sets of type-2 under algebraic product and algebraic sum," Fuzzy Sets and Systems, vol. 5, pp. 277$290,1981$.

[25] J. T. Starczewski, "A triangular type-2 fuzzy logic system," Proc FUZZ-IEEE 2006, Vancouver, B. C. Canada, pp. 7231-7238, July 2006.

[26] H. Tahayori, A. G. B. Tettamanzi and G. D. Antoni, "Approximated type-2 fuzzy set operations," Proc. FUZZ-IEEE 2006, Vancouver, B. C. Canada, pp. 9042-9049, July 2006.

[27] D. Wu and J. M. Mendel, "Enhanced Karnik-Mendel algorithms," accepted for publication in IEEE Trans. on Fuzzy Systems, 2007.

[28] H. Wu and J. M. Mendel, "Uncertainty bounds and their use in the design of interval type-2 fuzzy logic systems," IEEE Trans. on Fuzzy Systems, vol. 10, pp. 622-639, Oct. 2002.

[29] L. A. Zadeh, "The concept of a linguistic variable and its application to approximate reasoning-1," Information Sciences, vol. 8, pp. 199-249, 1975. 